
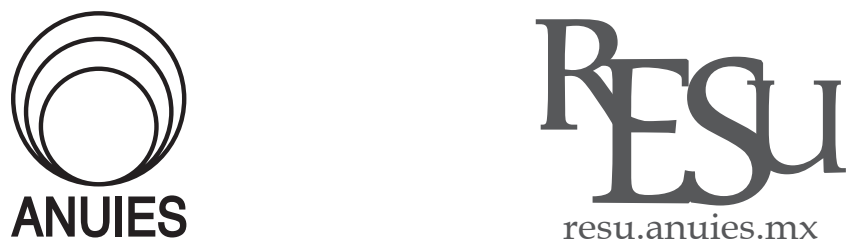

ARTíCULO

\title{
La Responsabilidad Social en la Educación Superior: Una revisión de la alineación entre el discurso educativo oficial y el curriculum explícito de las instituciones de educación superior de Oaxaca.
}

\section{Digital Social Responsibility in Higher Education:}

A review of the alignment between the official educational discourse and the explicit curriculum of Oaxaca's higher education institutions.

\author{
Vásquez Ibáñez Víctor * \\ * Centro de posgrado, Universidad Anáhuac Oaxaca, Oaxaca de Juárez, Oaxaca, México \\ Correo electrónico: vikofilosofo@gmail.com
}

Recibido el 17 de enero del 2018; aceptado el 16 de julio del 2019

\section{Resumen}

Este artículo analiza la alineación curricular, desde el concepto de Responsabilidad Social, entre el discurso oficial educativo de la autoridad correspondiente y el curriculum explícito de 81 instituciones de educación superior del estado de Oaxaca. Para lograr el objetivo planteado se utilizó un enfoque mixto apoyado en los principios metodológicos del análisis del discurso y el análisis del contenido. Se logró identificar la frecuencia con la que aparece el concepto y que el sentido denotado está más orientado a la responsabilidad individual y a la responsabilidad social en general, pero todavía distante de la responsabilidad social universitaria como categoría propiamente educativa.

Palabras clave: Educación superior, responsabilidad social, análisis del discurso, alineación curricular 


\begin{abstract}
This article analyzes the curricular alignment, after the concept of Social Responsibility, between the official educational discourse of the corresponding authority and the explicit curriculum of 81 Higher Education Institutions in the state of Oaxaca. In order to achieve this objective, a mixed approach was used, based on the methodological principles of discourse and content analysis. It was possible to identify the frequency -with which the concept appears- and that the sense denoted is generally more oriented to individual and social responsibility, but still far from university social concern as an appropriate educational category.
\end{abstract}

Keywords: Higher education, social responsibility, speech analysis, curriculum alignment

\title{
Introducción
}

A nte la eclosión de problemas sociales en la cotidianidad de las sociedades contemporáneas, la pregunta por la función de las Instituciones de Educación Superior (IES) es fundamental; aún más, el vínculo universidad-sociedad ha sido seriamente cuestionado desde las primeras décadas del siglo pasado (Ortega, 2015) y al inicio de éste (De Sousa, 2007). El análisis de esa relación no disminuye en nuestros días, al contrario, sigue replanteándose (Navarro y Villarroel, 2011, Fernández, 2014) y sobre todo pensándose a la luz de su pertinencia social (Leite y Beltrán, 2012).

En México la voz de los conflictos sociales resuena en los medios de comunicación que nos informan, día con día, aquello que bien podríamos parafrasear y denominar como el "mal de nuestro tiempo"; pero, de manera particular, el estado de Oaxaca vive una de sus peores crisis sociales, no sólo por los violentos acontecimientos ocurridos en el estado durante 2016, sino por lo que las estadísticas reflejan. Solamente dos referencias servirán para mostrar lo que está pasando: a) en primera instancia, el índice de rezago social aumentó constantemente durante los últimos quince años: 2.16090 (2000), 2.20393 (2005), 2.41779 (2010) 2.53901 (2015), provocando que Oaxaca pase al 1er lugar en rezago social dentro del contexto nacional (coneval, 2016: $10)$; b) en segunda instancia, el Instituto Nacional de Estadística y Geografía (INEGI) implementó un "visor dinámico de bienestar", que presenta no sólo el bienestar "objetivo", sino también el "subjetivo". El primero refiere los satisfactores materiales necesarios en una comunidad, pero el segundo refleja la experiencia de vida personal. El visor indica que el bienestar objetivo del 
oaxaqueño es el más bajo en el contexto nacional y la valoración del bienestar subjetivo indica que el promedio de satisfacción con la vida es de 7.5, el más bajo a nivel nacional (inegi, 2015), confirmando lo ordinario que se ha vuelto este ambiente, gestor lamentable de los problemas sociales en Oaxaca.

En el imaginario colectivo de la sociedad oaxaqueña, la falta de responsabilidad y compromiso social de los profesionales en activo se presenta como un factor que ha influido en su status actual, puntualizado por los índices mencionados en el párrafo anterior. Sin embargo, el juicio no sólo recae en los profesionales, sino también en aquellos que están en medio de su proceso de formación profesional, es decir, en los estudiantes de Instituciones de Educación Superior (IES). Se entiende que la falta de responsabilidad social de un profesionista está relacionada con la carencia de la misma en su educación superior, tal como lo muestran distintos estudios que ven una relación directamente proporcional entre la educación superior y la formación ciudadana o moral (Martínez, 2006; Guerrero y Gómez, 2013; Arango, Clavijo, Puerta y Sánchez, 2014).

Desde esta perspectiva, la relación entre la formación profesional desarrollada en las ies y los problemas sociales puede ser pensada desde un nuevo aspecto: la Responsabilidad Social (RS).

\section{Emergencia histórica de la Responsabilidad Social}

La literatura existente sobre el origen y la historia de la rs nos remonta a la mitad del siglo xx, cuando a través de su inclusión en el ámbito organizacional aparece formalmente en el panorama mundial. Algunos autores ubican el despunte de la responsabilidad social empresarial a finales de los años $50 \mathrm{y}$ principios de los 60 en Estados Unidos (Aguilera y Puerto, 2012) con Howard Bowen (Duque, Cardona y Rendón, 2013), sin embargo, Correa (2007) remonta su origen a finales del siglo XIX.

Si la empresa respondió a las exigencias actuales del escenario mundial, asumiendo la rs como enfoque de gestión, un poco más tarde, en el mismo siglo $\mathrm{xx}$, las IES no pudieron permanecer ajenas a los requerimientos globales y también empezaron a asumir la rs (Viteri, Jácome, Medina y Piloto, 2012).

La producción teórica sobre la Responsabilidad Social Universitaria (rsu) se sitúa a finales del siglo xx y principios del xxi, sin embargo se reconoce nuevamente a Bowen como uno de los primeros referentes, pues en 1984 incluye el concepto en su artículo "Graduate education and social responsibility", atribuyéndole dos aspectos: un ámbito técnico y otro moral (Fresán, 2012). 
La RS se presenta con mayor recurrencia en el ámbito educativo, cobrando autonomía semántica y pertinencia dentro del contexto universitario. El interés va desde la construcción de su marco teórico (Vallaeys, 2006), la identificación de enfoques o estrategias implementadas a nivel directivo (Olarte y Ríos, 2015), así como el atractivo social que despierta en la formación de un mejor ciudadano y, por ende, de una sociedad más justa (Arango, et al. 2014).

En México ya existen acciones serias encaminadas a la rs, como la creación del Observatorio Mexicano de Responsabilidad Social Universitaria (OMERSU) en el 2014, o la realización del segundo Congreso Internacional de Ética y Responsabilidad Social Universitaria organizado por la unam en mayo de este año, primer congreso de su tipo en México. Sin embargo, en Oaxaca todavía no se ha generado el interés que podría preverse si se toman en cuenta sus circunstancias político, sociales y culturales; solamente se han logrado identificar "eventos" muy esporádicos que podrían relacionarse indirectamente con la RS o la RSU.

En el Programa de Educación Superior de Oaxaca (peso) 2011-2016 aparece por primera vez, de manera explícita, sistemática y estratégica, la idea de la rs como un atributo de la educación superior (coepes, 2012: 7), recuperada desde las prioridades indicadas por la unesco en sus conferencias mundiales de 1998 y 2009, asumiendo como propia la idea y la importancia de la rs, plasmándola en el seno de la Filosofía de la Educación Superior en Oaxaca, especialmente en la enunciación de su misión, su visión y sus valores (coepes, 2012: 21).

El asumir y presentar, por vez primera, una postura pública de las autoridades en materia educativa respecto a la rs, es un momento crucial para entender el devenir histórico de la misma en la educación superior del estado de Oaxaca, y el punto de partida de la presente investigación, pues si la rs se presenta como uno de los ejes transversales de la educación superior y como uno de los principales valores que deben "vivirse" en este nivel, entonces es inevitable no preguntarse por la forma concreta en que esto se ha asumido por parte de las instituciones, cuestionando: ¿En qué porcentaje y en qué sentido se encuentran alineados, en términos de Responsabilidad Social, el discurso oficial de la autoridad educativa y el curriculum explícito de las Instituciones de Educación Superior del estado de Oaxaca?

\section{El discurso educativo}

El Discurso es un concepto polisémico (Sabaj, 2008 y López, 2015) tanto en su significado como en su uso; sin embargo, se identifican dos concepciones 
muy claras: a) como una manifestación lingüística, y b) como una manifestación más compleja, apelando "a sus relaciones contextuales, a sus implicaciones ideológicas, que se plasman en el artefacto discursivo" (Tarin, 2006, p. 8), y, por lo tanto, requiere no sólo de la lingüística, sino de varias disciplinas que lo atraviesen:

En un sentido amplio, el discurso es una práctica social, un evento comunicativo complejo que involucra a actores sociales, principalmente en sus roles de hablante/escribiente y oyente/lector. Éstos intervienen en un acto comunicativo, en una situación específica y son determinados por las características del contexto. (López, 2015: 27).

Si el discurso es una práctica social, entonces es lógico que esté presente en "todos lados", en la vida pública y la vida íntima de las personas (monólogos introspectivos), escapando así a la solitaria aproximación lingüística que normalmente le custodiaba. Sin embargo, se puede focalizar y clasificar el discurso, sobre todo atendiendo al contexto de su emisión y sus autores, aunque parece no haber consenso al respecto y se siguen buscando criterios más específicos, tal como lo hace Van Dijk cuando busca definir y diferenciar el discurso político del legal, el médico y el educativo (1999: 13).

Aquí emerge un hecho fundamental para esta investigación: tanto las prioridades de la unesco en sus conferencias mundiales, la filosofía de la educación superior en el peso 2011-2016, como el curriculum explícito de las IES en Oaxaca, se enuncian discursivamente, es decir, son un tipo de discurso, que por el contexto y la institución que los emite se identifican dentro del ámbito del discurso educativo, pues:

Un discurso educativo es una estructura que responde a un determinado objetivo educativo de la sociedad y que supone o establece un determinado lazo social estable, y que asigna lugares a los sujetos, lugares en la subjetividad, a los que también les asigna responsabilidades, formas de comportamiento y además les plantea una serie de exigencias, es decir, ciertos resultados. (Correa, 2009: 2-3)

Por lo tanto, el discurso proveniente de la coepes es educativo pero con matices políticos, mientras que el discurso emitido por las IES en su curriculum explícito es propiamente educativo. En los dos casos se tiene un discurso que "aparece como el portavoz de una serie de ideales, propósitos y políticas sociales, pero también de planteamientos teóricos que hallan sustento en presupuestos antropológicos" (Correa, 2009: 5). La diferencia radica en que en el primer caso tenemos un discurso pronunciado por un organismo descentralizado, no con el objetivo directo de educar (proceso de enseñanza- 
aprendizaje) sino de orientar a las instituciones encargadas de educar y de prescribir principios generales que orienten a la educación superior. En el segundo caso, el discurso es emitido con el fin de orientar el mismo acto educativo y la formación de los profesionales que se sujeten a dicho proceso.

\section{El curriculum explícito}

Al analizar la presencia del concepto de rs en el curriculum explícito, visto como discurso educativo, es necesario dilucidar el bagaje conceptual que implica entrar en el ámbito del diseño curricular. Para empezar, es válido precisar que cuando hablamos de curriculum, éste puede tener distintas clasificaciones: oficial, nulo, oculto y real (Posner, 2004), pero siguiendo a Casarini, (2012), el curriculum explícito u oficial es aquél que se presenta de forma escrita, por lo tanto, sintetiza formalmente el proceso de enseñanza-aprendizaje en el plan(es) de estudio(s) de la institución educativa.

Ahora bien, si se toma en cuenta que el plan de estudios busca "satisfacer las necesidades de una sociedad que requiere un profesional en un campo específico" (Roldan, 2005: 113), y además identificamos que una de sus relaciones significativas es la que establece con el contexto social (De Alba, 1985), entonces se dimensiona el valor que tiene el plan de estudios (presente como discurso) como concentrador de los fines e ideales de una institución educativa. Pero no sólo el plan de estudios contiene los principios educativos que rigen una institución, sino también la filosofía institucional que impacta en la vida cotidiana de los integrantes de la institución educativa (Münch, Galicia, y Jiménez, 2010).

A la luz de lo anterior, es adecuado el concepto de alineación curricular, "que es en general la congruencia que debe existir entre los diferentes elementos del currículo" (Alcoba, 2013: 242) para analizar el curriculum explícito, sin embargo, aunque hay autores que solamente la proponen en relación a lo que se enseña, cómo se enseña y cómo se evalúa (Biggs, 2006: 46), hay otros que ven la alineación como la concordancia entre los documentos normativos, los objetivos y la evaluación (Näsström y Henriksson, 2008). Pero también hay quien sí entiende que "el alineamiento curricular es una propiedad que opera a nivel de política educativa y del macro-sistema escolar" (Zúñiga, Arévalo y Maggio, 2014), y que si tomamos en cuenta, por lo tanto, que el curriculum tiene tres niveles de concreción (Romero, 2014: 203), a saber, macro, meso y micro (Rodríguez, Reyes y Peña, 2009), entonces se identifica que el discurso oficial de la autoridad educativa se sitúa en el nivel macro. La filosofía institucional y el plan de estudio están ubicados en el nivel meso, 
pues ya son competencia del centro educativo (Guzmán, Arreola, Martínez, y Solís, 2013).

Tomando en cuenta los elementos expuestos hasta aquí, el objetivo de este artículo es identificar la presencia y el sentido con el que se usa el concepto de Responsabilidad Social en el discurso oficial de la autoridad educativa y en el curriculum explícito de las IES de Oaxaca para determinar su grado de alineación curricular.

\section{Análisis del discurso como perspectiva teórico-metodológica}

En un primer momento se eligió el Análisis de Contenido (ac) ya que en el nivel informacional o referencial nos da la pauta para reconocer la presencia de la palabra (unidad de análisis) en el discurso, y en uno segundo se partió de orientaciones metodológicas del Análisis del Discurso (ad) (Urra, Muñoz y Peña, 2013). Aunque se reconoce su carácter transdisciplinar, tal como lo ha defendido van Dijk (2014), en esta investigación se utilizó como una técnica, que dicho sea de paso ha ganado mayor presencia en las Ciencias Sociales (Zaldua, 2006; Sayago, 2014) y también en las investigaciones educativas, desde el análisis del discurso de los profesores en el aula (Cárdenas y Rivera, 2006) y el análisis de la política educativa (Soler, 2011), conformando una perspectiva metodológica consolidada ya en México: el análisis político del discurso (Buenfil, 2010; López, Sañudo y Maggi, 2013).

Este estudio está apegado a la propuesta de análisis de la Gramática Sistémico Funcional de Halliday (Halliday 1994, Halliday y Hasan 1990), donde la unidad de análisis es la oración; pero lo particular de la gsf es que genera una atractiva y compleja propuesta de vinculación entre el texto y el contexto, reconoce la influencia del contexto en el discurso, identificando así el contexto de situación y el contexto de cultura.

Desde esta perspectiva se precisan tres dimensiones en todo contexto situacional (campo, modo y tenor) que se relacionan directamente con las tres metafunciones del lenguaje propuestas por Halliday (función ideativa, interpersonal e informativa); cada una de ellas es expresada gramaticalmente y por lo tanto susceptible de ser explicada por medio de recursos lingüísticos como la transitividad, el modo, el tema, entre otros.

La razón por la que se han elegido técnicas distintas pero complementarias, es debido a que el análisis de contenido implica una teoría simple que entiende el lenguaje como transparente, donde la palabra o frase representa el concepto que está detrás. Para el análisis del discurso la teoría es más com- 
pleja, desde la que se ve el lenguaje como un fenómeno mediado e influido por distintos factores; toma en cuenta el contexto en el que el hablante vive,

[...] un mundo donde el habla tiene efectos: donde no es indiferente referirse a alguien como 'soldado', 'guerrillero/a', 'terrorista' o 'luchador/a por la libertad'; o denominar y/o autodenominar a una organización como 'antiabortista' o 'pro vida' (Iñiguez y Antaki, 1998: 283).

\section{Metodología}

Esta investigación fue realizada desde un enfoque mixto y con un carácter descriptivo, pues, aunque se parte de un análisis cuantitativo, la pregunta de investigación presenta como intención última esclarecer la forma o manera (cualidad) en que se presenta la Rs en el discurso oficial, no solamente cuántas veces aparece sino, en última instancia, cómo aparece.

El método que se utilizó fue recuperado del análisis del discurso (ad) y complementado con el análisis de contenido (ac); cabe aclarar que fueron utilizados en la medida en que contribuyeron a determinar la presencia semántica de la Responsabilidad Social en el discurso y curriculum para identificar la probable alineación curricular, pero no con la pretensión de realizar el análisis lingüístico (exclusivamente) del discurso. En otras palabras, tuvieron una función instrumental, como medios para llegar a un fin que no se agotaba en la dilucidación lingüística.

Por lo tanto, se establecieron los siguientes momentos metodológicos:

1.Analizar el discurso oficial de la autoridad educativa.

Para ello se subdividió en dos momentos más:

a) Identificación del discurso:

- Se recuperó el texto del peso 2011-2016, pues es actualmente el único documento oficial en donde se hace mención explícita de la rs en la educación superior, emitido por la máxima autoridad en el área de educación superior en el estado de Oaxaca: la coepes.

- Se obtuvo el discurso oficial (oral) mediante una entrevista cualitativa a la persona que funge como informante clave en este estudio, a saber, el secretario técnico de la coepes, que además fue el coordinador general del peso 2011-2016. La entrevista fue efectuada desde su función como representante oficial de dicha comisión, evitando con ello posturas personales respecto al tema de investigación; la grabación resultante fue posteriormente transcrita para su análisis. La entrevista permite que el entrevistado 
comparta oralmente con el investigador aquello concerniente a un tema específico y del tipo semiestructurada porque permite un mayor grado de flexibilidad e interpretación (Torruco, Díaz, Varela y Martínez, 2013).

b) Análisis del discurso:

- En un primer momento se procedió a realizar el análisis desde el nivel conceptual; para ello se fijó la palabra como unidad de análisis y se identificó la frecuencia de la categoría de rs y de categorías próximas a ella, las cuales fueron elegidas bajo los siguientes criterios: campo semántico, familia léxica, sinonimia, antinomia y proximidad categorial.

- Para realizar el análisis cuantitativo del discurso se utilizó el software qda Miner y el WordStat, para determinar la frecuencia, la concurrencia y referencias cruzadas.

- Posteriormente se identificó su estructura interna, se aplicaron los principios de la gsf para introducir el análisis en un nivel interpretativo, se determinó su contexto cultural y el situacional a partir de las categorías de campo, tenor y modo.

2. Analizar el discurso educativo declarado en el curriculum explicito de las IES de Oaxaca:

- Identificación del discurso (curriculum explícito)

- Siguiendo al Programa Sectorial de Educación, la educación superior tiene por objetivo formar profesionales (sep, 2013: 104), entonces es en las IES en donde se juega dicha misión. Por lo tanto, se perfiló la muestra, siguiendo los principios establecidos por Bonilla y Rodríguez (2005) para el muestreo cualitativo, así que utilizando una combinación de muestra por criterio lógico y teórico se generó lo siguiente: de todas las IEs que hay en el estado, inicialmente se seleccionaron las que aparecían en los últimos catálogos de licenciaturas y posgrado de la coepes; de esta población inicial se filtraron solamente aquellas instituciones que tiene oferta a nivel licenciatura, resultando entonces un total de 81 , mismas que han sido declaradas y presentadas en su Catálogo de Licenciaturas 2016-2017

- Se procedió a recoger los datos a través de la investigación documental, extrayendo los elementos requeridos de dos fuentes: los catálogos de la coepes (2015 y 2016) y la página web de cada institución.

La validez y la confiabilidad de los datos, desde las concepciones propias a la investigación cualitativa, son generadas por las siguientes razones: a) la coepes en su calidad de autoridad máxima en educación superior envía cada año la invitación a las IES para actualizar y registrar su oferta educativa; b) las IES aceptan voluntariamente enviar o no su información, pero si lo hacen el requerimiento de la coepes es que sea la información oficial y actualizada 
de cada institución; c) La coepes realiza un proceso de revisión interna, a través de los formatos 911 y de la base de datos de la Subsecretaría de Educación Superior, para validar la información proporcionada por cada IES. De tal manera que la información contenida en los catálogos, incluida la página web, es información declarada como oficial por los informantes (muestra) y valorada por la autoridad correspondiente.

Una vez establecida la validez de la fuente, se transcribió la filosofía institucional y plan de estudios, respetando su originalidad textual, pero se modificó su forma de presentación, ya que se colocaron en un formato diseñado para facilitar su tratamiento en el programa informático seleccionado para analizarlos (qda Miner).

- Análisis del curriculum explícito como discurso.

- Se utilizaron las mismas categorías que en el discurso oficial y se organizaron los elementos del curriculum que se analizarían; para ello se utilizó la matriz de "alineación curricular macro-meso", que se derivó del instrumento "matriz de coherencia curricular" (Castañeda, Castro y Mena, 2012: 78), construida en torno a los siguientes elementos del curriculum explícito:

a. Filosofía institucional: misión, visión y valores,

b. Plan (es) de estudio (s): objetivo de carrera, perfil de egreso y mapa curricular.

- Se procedió a realizar el análisis desde el nivel conceptual; para ello se fijó a la palabra como unidad de análisis y se identificó la frecuencia de las categorías; después se concentró el análisis en "responsabilidad" y "responsabilidad social", por ser las que mayor relación tienen con el objetivo planteado. Se volvió a focalizar el análisis, ahora solamente con la categoría principal, tomándose como criterios la exactitud morfológica de la palabra y que denotara su relación con la sociedad.

- Después se seleccionó la misión de la institución que contiene explícitamente la categoría de responsabilidad social; se le recuperó en su contexto original y se le aplicaron los principios de la gsf para introducir nuevamente el análisis en un nivel interpretativo.

3. Con los resultados obtenidos del análisis anterior se pudo obtener el porcentaje de alineamiento de las IES, ya que se le asignó el valor de 1 a la presencia y 0 a la ausencia de la categoría, tanto en la filosofía como en el plan de estudios de cada institución.

Dando un paso más en el nivel de análisis, se escogieron a cuatro IES, las primeras tres porque son las que mayor frecuencia tienen de las categorías "responsabilidad" y "responsabilidad social"; y la cuarta (a) por ser la única institución que utiliza la categoría de "responsabilidad social" en su misión 
institucional, la cual fue analizada con las categorías de la gsf del contexto cultural y situacional.

Se estudió cada uno de los elementos del curriculum explícito a la luz de dos criterios: uso del concepto de "responsabilidad social" y la semejanza del sentido de "responsabilidad social" en su enunciación con la del discurso oficial, ayudándonos nuevamente de las categorías de análisis de la gsf.

Finalmente, se analizaron dos segmentos de los dos discursos: la misión del peso 2011-2016 y la misión de la institución A, porque coinciden en ser las únicas "misiones" que integran la categoría principal de la investigación y se analizó el sentido con el que usan el concepto de "responsabilidad social" y la relación que hay entre ellas.

\section{Resultados}

\section{Discurso oficial - Nivel Conceptual (ac)}

En el análisis que se realizó al discurso oficial en el nivel conceptual, es decir, cuantitativo, muestra una sutil presencia de las categorías buscadas; la mayoría de ellas no están presentes en el documento.

El primer documento contiene un total de 14, 453 palabras, de la cuales solamente "igualdad", "servicio", "beneficio", "responsabilidad", "responsable", "corresponsabilidad", "compromiso" y "necesidad" tuvieron una relevancia para los objetivos del estudio, pues en el contexto en que se presentan normalmente aparecen acompañadas del adjetivo "social", sin embargo, su frecuencia es mínima.

En el segundo documento el resultado resultó evidentemente distinto, pues el discurso analizado fue producto de una entrevista con preguntas claramente dirigidas al tema de la responsabilidad social y referidas al primer documento, sin embargo, el corpus fue constituido únicamente por las respuestas del informante clave. En la siguiente tabla se pueden visualizar los resultados:

\begin{tabular}{|c|c|c|}
\hline \multirow{3}{*}{ Categorías } & \multicolumn{2}{|c|}{ Discurso oficial (coepes) } \\
\hline & Corpus $1(14,453$ palabras $)$ & Corpus $2(3,821$ palabras) \\
\hline & \multicolumn{2}{|c|}{ Frecuencia } \\
\hline Igualdad (es) & $2(0.01 \%)[\mathrm{fi}=2 / \mathrm{hi}=0.00013]$ & $0(0.00 \%)[\mathrm{fi}=0 / \mathrm{hi}=0.00000]$ \\
\hline Igualdad (es) "social” (es) & $2(0.01 \%)[\mathrm{fi}=2 / \mathrm{hi}=0.00013]$ & $0(0.00 \%)[\mathrm{fi}=0 / \mathrm{hi}=0.00000]$ \\
\hline Servicio & $11(0.08 \%)[\mathrm{fi}=11 / \mathrm{hi}=0.00076]$ & $1(0.03 \%)[\mathrm{fi}=1 / \mathrm{hi}=0.00026]$ \\
\hline
\end{tabular}




\begin{tabular}{|c|c|c|}
\hline \multirow{3}{*}{ Categorías } & \multicolumn{2}{|c|}{ Discurso oficial (coepes) } \\
\hline & Corpus $1(14,453$ palabras $)$ & Corpus $2(3,821$ palabras) \\
\hline & \multicolumn{2}{|c|}{ Frecuencia } \\
\hline Servicio "social" & $9(0.06 \%)[\mathrm{fi}=9 / \mathrm{hi}=0.00062]$ & $1(0.03 \%)[\mathrm{fi}=1 / \mathrm{hi}=0.00026]$ \\
\hline Beneficio(s) & $5(0.03 \%)$ [fi=5 / hi=0.00034] & $0(0.00 \%)$ [fi $=0 / \mathrm{hi}=0.00000]$ \\
\hline Beneficio(s) “social” (es) & $4(0.03 \%)[\mathrm{fi}=4 / \mathrm{hi}=0.00027]$ & $0(0.00 \%)[\mathrm{fi}=0 / \mathrm{hi}=0.00000]$ \\
\hline Responsabilidad & $8(0.06 \%)[\mathrm{fi}=8 / \mathrm{hi}=0.00055]$ & $15(0.46 \%)$ [fi $=15 / \mathrm{hi}=0.00392]$ \\
\hline Responsabilidad "social" & $2(0.01 \%)[\mathrm{fi}=2 / \mathrm{hi}=0.00013]$ & $14(0.43 \%)[\mathrm{fi}=14 / \mathrm{hi}=0.00366]$ \\
\hline Responsable (s) & $2(0.01 \%)[\mathrm{fi}=2 / \mathrm{hi}=0.00013]$ & $3(0.09 \%)[\mathrm{fi}=3 / \mathrm{hi}=0.00078]$ \\
\hline Corresponsabilidad & $1(0.01 \%)[\mathrm{fi}=1 / \mathrm{hi}=0.00006]$ & $0(0.00 \%)[\mathrm{fi}=0 / \mathrm{hi}=0.00000]$ \\
\hline Compromiso & $3(0.02 \%)[\mathrm{fi}=3 / \mathrm{hi}=0.00020]$ & $1(0.03 \%)[\mathrm{fi}=1 / \mathrm{hi}=0.00026]$ \\
\hline Compromiso "social" & $2(0.01 \%)[\mathrm{fi}=2 / \mathrm{hi}=0.00013]$ & $1(0.03 \%)[\mathrm{fi}=1 / \mathrm{hi}=0.00026]$ \\
\hline Necesidad (es) & $12(0.08 \%)[\mathrm{fi}=12 / \mathrm{hi}=0.00083]$ & $2(0.06 \%)[\mathrm{fi}=2 / \mathrm{hi}=0.00052]$ \\
\hline Necesidad (es) "social” (es) & $9(0.06 \%)[\mathrm{fi}=9 / \mathrm{hi}=0.00062]$ & $1(0.03 \%)[\mathrm{fi}=1 / \mathrm{hi}=0.00026]$ \\
\hline
\end{tabular}

Como se puede observar, la categoría "responsabilidad social" se encuentra presente en los dos corpus, y la frecuencia con la que aparece en los dos es mínima, pues si se toma en cuenta la frecuencia absoluta de otro tipo de categorías como "educación" o "superior", resulta evidente que ninguno de los dos documentos se caracteriza por la presencia abundante de la rs. Por el contrario, tomando en cuenta estos resultados, debería descartarse el documento por no tener una carga léxica considerable en torno al concepto de RS. El análisis conceptual resulta así, y por sí solo, insuficiente. Sin embargo, no es la cantidad de veces que aparece la categoría lo que resuelve completamente la pregunta de investigación; se requiere pasar al siguiente nivel de análisis para dilucidar su significado.

\section{Discurso oficial - Nivel interpretativo (ad - gsf)}

Se analizó la estructura y el contexto del peso 2011-2016, así como el corpus resultante de la entrevista realizada al Secretario Técnico de la coepes.

Para la gsf, los textos son productos concretos de un determinado contexto social y, por lo tanto, también son resultado de las elecciones - para nada arbitrarias - que realiza el emisor, adaptadas evidentemente al contexto (cultural y situacional) y a la función que tendrá el texto.

Una vez aplicados los principios de la gsf a cada uno de los corpus, se encontró lo siguiente: 


\section{Corpus 1}

- Contexto cultural: El peso 2011-2016 es formulado y publicado en un momento histórico clave de Oaxaca, pues por primera vez había ganado la gubernatura un representante de la oposición, terminando así el reinado, de más de sesenta años, del Partido Revolucionario Institucional. Evidentemente que la sociedad y el estado de Oaxaca vivían un ambiente de renovación política y de esperanza en el desarrollo económico-social; entre el año 2010 y 2012 se conformó el discurso que finalmente fue publicado y puesto a disposición de las IES y de todo aquel que quisiera consultarlo en el 2012.

No tenía más de dos años que había sucedido la Conferencia Mundial de Educación Superior de la unesco en el año 2009, la cual se convertió en pilar fundamental de lo enunciado en la filosofía de la educación superior de Oaxaca y eso quedó constatado por un alineamiento entre las finalidades de la unesco y las de la coepes, situación que fue corroborada por el informante clave, pues como él dice "[...] para poder pensar localmente, debemos pensar globalmente". (Entrevista-ST, 2016).

\section{Corpus 2}

- Contexto cultural: La entrevista oficial que se realizó al Secretario Técnico ocurrió en un momento clave, pues fue precisamente en el último año que tuvo vigencia el peso 2011-2016, es decir, si había un momento para evaluar los alcances dicho programa, tenía que ser entonces..

El informante clave juega un papel fundamental, pues coordinó el proceso de elaboración del peso 2011-2016 y actualmente funge como Secretario Técnico de la comisión que lo construyó y publicó, es decir, si hay alguien que ha estado en todo el proceso de lo que implica ese discurso, ha sido justamente él.

Así como en el 2010 se vivía un ambiente de cambio, en el 2016 se percibió en la sociedad un "regresar a lo mismo", sobre todo después de los conflictos sociales y políticos que acontecieron en este año, por esto, no resulta extraño que para la coepes se siga considerando el tema de la rs en las IES - y en general de todos los actores educativos - como un aspecto fundamental para la educación superior. 
- Contexto situacional:

\begin{tabular}{|c|c|c|}
\hline \multirow{2}{*}{ Categorías } & \multicolumn{2}{|c|}{ Discurso oficial (coepes) } \\
\hline & peso $2011-2016$ & Entrevista Oficial \\
\hline 1. Campo & $\begin{array}{l}\text { Orientar respecto a las finalidades de la Educa- } \\
\text { ción Superior en el estado de Oaxaca durante el } \\
\text { sexenio gubernamental 2010-2017 }\end{array}$ & $\begin{array}{l}\text { Aclarar el contexto en } \\
\text { que se produjo el peso } \\
\text { 2011-2016 }\end{array}$ \\
\hline 2. Tenor & $\begin{array}{l}\text { coepes-IES } \\
\text { La coepes es la emisora, en común } \\
\text { acuerdo con los representantes de las } \\
\text { IES y su audiencia directa son las mis- } \\
\text { mas IES e indirectamente el Gobierno } \\
\text { del Estado y finalmente la sociedad. }\end{array}$ & $\begin{array}{l}\text { Secretario Técnico- Entre- } \\
\text { vistador } \\
\text { El secretario técnico es el } \\
\text { emisor y su audiencia es el } \\
\text { investigador. }\end{array}$ \\
\hline & Comisión-Afiliados & Entrevistado-Entrevistador \\
\hline 2.1. Poder & Igualdad & $\begin{array}{l}\text { Experto - aprendiz (poder } \\
\text { intelectual) }\end{array}$ \\
\hline 2.2. Contacto & Frecuente (colaborativo) & Frecuente (personal) \\
\hline 2.3. Implicación afectiva & Nula & Media (empatía) \\
\hline 3. Modo & Preparado y pensando con tiempo & Preparado previamente \\
\hline 3.1 Canal & Escrito-digital & Oral-escrito \\
\hline 3.2 Género & Género informativo-prescriptivo & Género informativo-explicativ \\
\hline
\end{tabular}

\section{Curriculum explícito - Nivel Conceptual (ac)}

El primer nivel de análisis que se realizó al curriculum explícito fue también conceptual, por lo tanto se partió de las mismas categorías a priori que en el análisis del discurso oficial. Los resultados fueron los siguientes:

\begin{tabular}{lc}
\hline \multirow{2}{*}{ Categorías } & $\begin{array}{c}\text { Discurso educativo (curriculum explícito) } \\
\mathbf{1 7 5}, \mathbf{8 0 1} \text { palabras }\end{array}$ \\
\cline { 2 - 2 } Igualdad (es) & Frecuencia \\
\hline Igualdad (es) "social" (es) & $6(0.00 \%)[\mathrm{fi}=6 / \mathrm{hi}=0.00003]$ \\
\hline Servicio & $0(0.00 \%)[\mathrm{fi}=0 / \mathrm{hi}=0.00000]$ \\
\hline Servicio "social" & $179(0.10 \%)[\mathrm{fi}=179 / \mathrm{hi}=0.00101]$ \\
\hline Beneficio(s) & $64(0.04 \%)[\mathrm{fi}=55 / \mathrm{hi}=0.00036]$ \\
\hline Beneficio(s) "social" (es) & $29(0.02 \%)[\mathrm{fi}=29 / \mathrm{hi}=0.00016]$. \\
\hline Responsabilidad & $27(0.01 \%)[\mathrm{fi}=27 / \mathrm{hi}=0.00015]$ \\
\hline Responsabilidad "social" & $173(0.10 \%)[\mathrm{fi}=173 / \mathrm{hi}=0.00098$ \\
\hline
\end{tabular}




\begin{tabular}{lc}
\hline \multirow{2}{*}{ Categorías } & Discurso educativo (curriculum explícito) \\
& $\mathbf{1 7 5}, \mathbf{8 0 1}$ palabras \\
\cline { 2 - 2 } Responsable (s) & Frecuencia \\
\hline Corresponsabilidad & $29(0.02 \%)[\mathrm{fi}=29$ / hi $=0.00016]$ \\
\hline Compromiso & $5(0.00 \%)[\mathrm{fi}=5 / \mathrm{hi}=0.00002]$ \\
\hline Compromiso "social” & $129(0.07 \%)[\mathrm{fi}=129 / \mathrm{hi}=0.00073]$ \\
\hline Necesidad (es) & $49(0.07 \%)[\mathrm{fi}=49 / \mathrm{hi}=0.00027]$ \\
\hline Necesidad (es) “social” (es) & $205(0.13 \%)[\mathrm{fi}=214 / \mathrm{hi}=0.00116]$ \\
\hline
\end{tabular}

En esta ocasión, la categoría "responsabilidad" se encuentra presente de una manera más constante en el corpus, pues aparece indiferenciadamente en 173 ocasiones; de ellas, 71 lo hace como valor o concepto individual, es decir, solamente como responsabilidad y en 102 ocasiones aparece denotando su relación con la sociedad, es decir, como "responsabilidad social", de las cuales sólo en 100 ocasiones aparece con exactitud morfológica.

De hecho, la categoría "responsabilidad social" aparece denotando su relación con la sociedad en 33 casos, es decir, en 33 IES y con exactitud morfológica en 31 de las 81 instituciones.

Como "responsabilidad" no aparece en ninguna misión de la filosofía institucional de las 81 IEs y como "responsabilidad social" sólo hace presencia en una con exactitud morfológica, y en una más denotando su sentido social. En el caso de la visión institucional, el panorama no es muy alentador, pues "responsabilidad" sólo aparece en 2 IES y como "responsabilidad social" en ninguna; por el contrario, "responsabilidad" aparece como valor institucional en 15 ocasiones y "responsabilidad social" en 2. Estos datos son significativos, ya que la forma en que aparecen estas dos categorías en el discurso oficial, es justamente dentro de la misión de la educación superior de Oaxaca y como uno de los valores a vivir por los agentes educativos.

\section{Curriculum explícito - Nivel interpretativo (ad - gsf)}

A diferencia del análisis que se realizó del discurso oficial, en el corpus conformado por el curriculum explícito no se tiene un texto homogéneo, a pesar de ello, se seleccionó la misión de la filosofía institucional de la única institución (A) que contiene la categoría con exactitud morfológica y denota evidentemente su sentido social, como se explicará más adelante, y recuperamos el contexto en el que se encontró para realizar un análisis paralelo al del discurso oficial. 
Aplicando nuevamente los principios de la GSF, notamos que el contexto cultural y situacional son similares a los contextos del discurso oficial; los dos se desarrollan en el ámbito educativo-político, están insertos en la sociedad de Oaxaca, con vigencia actual, con la finalidad de informar una situación específica de la educación superior, en un caso el estatus, en el otro la oferta. Tanto discurso oficial como curriculum explícito son emitidos por una autoridad educativa.

Para mejor sistematización y comparación, la misión enunciada vía catálogo de oferta es el corpus 1 y la misma misión enunciada vía página web es el corpus 2 .

\section{Corpus 1}

- Contexto cultural: La misión (curriculum explícito) está presentada en el Catálogo de Oferta de Posgrado en el Estado de Oaxaca 2015-2016; este documento y la institución emisora (coepes) fungen como intermediarios, entre el discurso de la institución educativa y el receptor potencial. Cabe destacar que dicho catálogo lleva realizándose durante los últimos seis años (2010-2016), como una iniciativa tanto de coepes como de las IES y la CGEMSySCyT, convirtiéndose en el mayor referente informativo para aquellas personas que pretenden estudiar un posgrado en el estado. La presentación que coepes hace de los datos de las instituciones es homogénea, utiliza los mismo recursos lingüísticos y gráficos para presentarlos, eliminando por completo algún tipo de tendencia que resalte a una institución por encima de otra.

El catálogo es publicado y distribuido a las IES integrantes de la misma coepes y a toda aquella persona interesada, por lo tanto tiene un carácter público.

\section{Corpus 2}

- Contexto cultural: La misión es presentada en la página web de la institución. En este caso sí se puede afirmar que la institución es la emisora y de forma directa hace llegar su discurso al receptor potencial; tiene los derechos reservados, y con ello, los de autoría de lo que ahí se publica. La página web es fácil de localizar, pues su link está conformado por las siglas de la universidad, el dominio edu y el país de origen $m x$, por lo tanto debe ser accesible a toda aquella persona que quiera consultarla, conforme tiene un carácter público. 
La misión no se encuentra inmediatamente en la primera pantalla que muestra la página web, por lo tanto hay que dar clic a la pestaña identidad - se encuentra en la parte inferior de la pantalla - que sí es visible de forma inmediata; por lo tanto, en la segunda pantalla aparece la misión.

- Contexto situacional:

\begin{tabular}{|c|c|c|}
\hline \multirow{2}{*}{ Categorías } & \multicolumn{2}{|c|}{ Discurso oficial (coepes) } \\
\hline & $\begin{array}{l}\text { Catálogo de Oferta de } \\
\text { Posgrado 2015-2016 }\end{array}$ & Página web \\
\hline 1. Campo & $\begin{array}{l}\text { Informar a los egresados de licencia- } \\
\text { tura, profesionistas y a la comunidad } \\
\text { académica en general la oferta de } \\
\text { posgrado en el estado de Oaxaca. }\end{array}$ & $\begin{array}{l}\text { Informar a los egresados de nivel } \\
\text { medio superior quién es, qué tiene, } \\
\text { que hace y qué ofrece la Institución de } \\
\text { Educación Superior (A) }\end{array}$ \\
\hline 2. Tenor & $\begin{array}{l}\text { coepes-Egresado del nivel profesional } \\
\text { La coepes es la emisora intermediaria, } \\
\text { pues informa lo que las IEs informan. } \\
\text { Comisión-matrícula potencial }\end{array}$ & $\begin{array}{l}\text { Institución A-público en general } \\
\text { La institución A es la emisora directa. } \\
\text { Institución-matricula potencial }\end{array}$ \\
\hline 2.1. Poder & $\begin{array}{l}\text { Desigual } \\
\text { Organo concentrador-aspirante }\end{array}$ & $\begin{array}{l}\text { Desigual } \\
\text { Institución ofertante-aspirante }\end{array}$ \\
\hline 2.2. Contacto & Esporádico (visual-manual) & Frecuente (visual-digital) \\
\hline 2.3. Implicación afectiva & Baja & Baja \\
\hline 3. Modo & Preparado y pensando con tiempo & Preparado previamente \\
\hline 3.1 Canal & Escrito-impreso & Escrito-digital \\
\hline 3.1 Género & Género informativo & Género informativo \\
\hline
\end{tabular}

\section{Alineación curricular a partir del discurso}

Desde el nivel de análisis conceptual (ac) y atendiendo a la categoría principal "responsabilidad social", la filosofía institucional de las 81 IEs del estado de Oaxaca, registradas por coepes, tienen un porcentaje mínimo de alineación: en la misión hay un $2.4 \%$, en la visión $0 \%$ y en los valores $2.4 \%$.

Por otra parte, aun cuando su frecuencia y su porcentaje de alineación son mínimos en la filosofía institucional, en el caso del plan de estudios, los resultados son los siguientes: como parte de los objetivos de la carrera aparece en 31 ocasiones, y tomando el total de objetivos por carrera analizados, su porcentaje de alineación es de $6.8 \%$; en los perfiles de egreso aparece 38 ocasiones, con $9.1 \%$ de alineación, y en la malla curricular 29, con $6.3 \%$ de alineación. 
Así, de las dos áreas del curriculum explícito que se analizaron, es en el plan de estudios donde hay mayor presencia del concepto, lo cual es digno de llamar la atención, pues esta área es más operativa que la filosofía institucional, es decir, en el objetivo de carrera, el perfil de egreso y las asignaturas se concreta lo declarado en la filosofía institucional.

Una vez establecido que la aparición de la categoría en el discurso, de cada uno de los elementos del curriculum explícito, es el punto de partida para realizar este nivel de análisis, entonces se puede determinar un porcentaje de alineación general para las 81 Instituciones de Educación Superior del estado de Oaxaca: $11.8 \%$ en relación a la categoría de "responsabilidad" en general y $7.0 \%$ en relación a la "responsabilidad social".

Al utilizar el análisis de contenido en el corpus creado por el curriculum explícito de las IES, se pudo constatar, como se ha visto, que la categoría principal de esta investigación sí está presente: la frecuencia con la que aparece e incluso identificar en qué casos se hace presente, cumpliéndose así parte del objetivo de investigación.

Ahora bien, los resultados del análisis de las cuatro instituciones escogidas por la presencia y la importancia que le dan a la Rs en su discurso los podemos visualizar en la siguiente tabla:

\begin{tabular}{|c|c|c|c|c|c|c|c|}
\hline \multirow[b]{2}{*}{$\begin{array}{l}\text { Nivel de } \\
\text { análisis }\end{array}$} & \multicolumn{3}{|c|}{ Filosofía institucional } & \multicolumn{3}{|c|}{ Plan de estudios } & \multirow[b]{2}{*}{$\begin{array}{c}\% \text { de } \\
\text { alineación }\end{array}$} \\
\hline & Misión & Visión & Valores & $\begin{array}{c}\text { Objetivo } \\
\text { de } \\
\text { carrera }\end{array}$ & $\begin{array}{c}\text { Perfil } \\
\text { de } \\
\text { egreso }\end{array}$ & $\begin{array}{c}\text { Malla } \\
\text { Curricular }\end{array}$ & \\
\hline \multicolumn{8}{|c|}{ Institución 1} \\
\hline Conceptual & $0 / 1$ & $0 / 1$ & $0 / 1$ & $1 / 14$ & $3 / 14$ & $14 / 14$ & $40 \%$ \\
\hline Interpretativo & $1 / 1$ & $1 / 1$ & $1 / 1$ & $13 / 14$ & $6 / 14$ & $14 / 14$ & $80 \%$ \\
\hline \multicolumn{8}{|c|}{ Institución 2} \\
\hline Conceptual & $0 / 1$ & $0 / 1$ & $0 / 1$ & $1 / 19$ & $2 / 19$ & $0 / 19$ & $5 \%$ \\
\hline Interpretativo & $1 / 1$ & $1 / 1$ & $1 / 1$ & $12 / 19$ & $16 / 19$ & $19 / 19$ & $83 \%$ \\
\hline \multicolumn{8}{|c|}{ Institución 3} \\
\hline Conceptual & $0 / 1$ & $0 / 1$ & $1 / 1$ & $1 / 9$ & $4 / 9$ & $0 / 9$ & $20 \%$ \\
\hline Interpretativo & $1 / 1$ & $1 / 1$ & $1 / 1$ & $4 / 9$ & $7 / 9$ & $5 / 9$ & $63 \%$ \\
\hline \multicolumn{8}{|c|}{ Institución A } \\
\hline Conceptual & $1 / 1$ & $0 / 1$ & $1 / 1$ & $1 / 13$ & $0 / 13$ & $0 / 13$ & $7 \%$ \\
\hline Interpretativo & $1 / 1$ & $1 / 1$ & $1 / 1$ & $4 / 13$ & $3 / 13$ & $8 / 13$ & $42 \%$ \\
\hline
\end{tabular}

Como se puede observar en estas cuatro instituciones representativas, hay un aumento considerable en el porcentaje de alineación cuando el nivel de 
análisis es interpretativo, es decir, se encontró que hay una mayor alineación cuando se busca el sentido de la responsabilidad social. Se reconocen aspectos de ésta como: desarrollo sustentable, bienestar de la comunidad, bien común, beneficio social, responsabilidad ambiental, ética profesional, entre otros; sin embargo, hay una menor alineación cuando se busca solamente el uso del concepto.

Parece ser que las IEs han entendido la necesidad de una oferta educativa con un sentido de responsabilidad social y lo reflejan en los conceptos que utilizan en su curriculum explícito, sin embargo, todavía no hay una alineación conceptual, pues no hay integración de la categoría "responsabilidad social" en el discurso que usan en su curriculum.

Finalmente, al analizar los dos segmentos seleccionados del discurso: la misión del peso 2011-2016 y la misión de la institución A, se encontró una semejanza significativa bajo las mismas categorías de campo, tenor y modo; además, las dos tiene una estructura similar dentro del discurso en que se encuentran, pues son tituladas como "misión". Se constituyen del título y un párrafo; los párrafos contienen 55 y 54 palabras respectivamente, las dos inician con un verbo en infinitivo y las dos tienen una tendencia a "ofrecer" la presencia de la categoría "responsabilidad social" al final del párrafo, mostrando con ello que funciona como complemento. Evidentemente no es el concepto fundamental de la misión en ninguno de los dos casos, sin embargo, por la posición y la forma de enunciación, es lo que le da sentido a la idea principal del párrafo. Tienen ideas en común como la formación de personas, calidad y pertinencia de la oferta. La población a la que se dirigen es la misma (sociedad oaxaqueña), pero sobre todo tienen continuidad en el sentido proyectado, es decir, en la misión de la educación superior promulgada por la coepes. Se presenta la responsabilidad social como dadora de sentido en la educación superior de Oaxaca, la cual debe ser asumida por las IES en la oferta que ponen a disposición de la sociedad, y esto es reflejado en la misión de la institución A, pues ya no se refiere a la educación superior en general, sino a su oferta educativa. Se presenta como aquel tipo de institución que describe la primera misión, de tal manera que la "responsabilidad social" no sólo es proveedora de sentido, sino una actitud (vivida) por los profesionistas que egresen de esta institución.

\section{Conclusiones}

A partir de los resultados obtenidos, se puede concluir que el porcentaje de alineación curricular entre el discurso oficial de la coepes y el curriculum ex- 
plícito de las IES del estado de Oaxaca es mínimo después de los cuatro años en los que ha estado vigente la misión construida para la educación superior. Sigue siendo una tarea pendiente para todas las instituciones el alinear conceptualmente el discurso educativo del curriculum explícito que presentan a la sociedad, para que se vea reflejada esa preocupación por formar profesionales con un sentido de responsabilidad social.

El hecho de que exista un sentido de responsabilidad social implícito en el curriculum es un paso hacia la integración del ideal promovido por el discurso oficial, pero sin lugar a dudas falta hacerlo explícito, y no por una simple necesidad de alinearse, sino porque, como se ha dicho al inicio de este artículo, es una necesidad social en el estado. En la medida que las IEs hagan suyo este concepto y lo reflejen en su curriculum explícito, habrá indicadores para que sus procesos formativos vayan encaminados hacia la adquisición de todas las competencias propias de cada profesión, pero siempre con un sentido de responsabilidad social.

De los dos elementos analizados del curriculum explícito, se identificó el plan de estudios como el más operativo, y en ese sentido es fundamental que las instituciones declaren en el perfil de egreso la actitud adquirida de la responsabilidad social. Además, una gran área de oportunidad se da en la malla curricular, pues como ya sucede con algunas de las instituciones analizadas, se puede integrar una asignatura que todo estudiante universitario deberá cursar, si bien es cierto que se logró identificar con mayor frecuencia la presencia de una asignatura relacionada con la ética profesional. Podría complementarse con otra referida a la formación directa de la responsabilidad social del futuro profesional.

Evidentemente que el límite de esta investigación está en el nivel mismo del curriculum y desde ahí se puede juzgar lo que está pasando. Bajar al nivel micro requeriría de otra investigación y revisar si en el quehacer cotidiano de la institución - a nivel aula de clases, por ejemplo - se está formando ese sentido de responsabilidad social a través de las planeaciones docentes, de programas institucionales, de eventos académicos o de espacios en la vida universitaria que sean generados con tal objetivo.

Por otra parte, el sentido con el que es usado el concepto está más cercano a la responsabilidad social empresarial que a la categoría emergente en las últimas décadas de responsabilidad social universitaria, ya que, por ejemplo, las carreras en que mayor presencia tiene el concepto son: administración de empresas, gestión empresarial, contaduría y derecho. No aparece de manera transversal en todas las carreras. A la luz de la perspectiva teórica de Vallaeys (2006), la rsu deberá impregnar de sentido a cada una de las dimensiones de la universidad o institución de educación superior, pero, de hecho, el 
concepto de RSU no aparece en ninguno de los corpus analizados, eso quiere decir que ni en la autoridad educativa oficial ni en las instituciones de educación superior hay todavía conciencia explícita de tal actitud.

Si ocurre en la educación superior del estado de Oaxaca como ha pasado en la evolución histórica del concepto, entonces se encuentra en una fase previa, que va en camino pero todavía no tiene claro por dónde caminar exactamente para formar profesionales con un alto sentido de responsabilidad social, que contribuyan al desarrollo de la sociedad oaxaqueña. Desde la coepes esta situación es evidente, por ejemplo, cuando comenta su Secretario Técnico que se ha fijado el criterio de responsabilidad social para valorar la pertinencia de los nuevos planes y programas de estudio. Pero también es consciente de que hacer operativo dicho concepto ha sido complicado, pues a la mayoría de las IES y a la misma comisión no les queda claro el concepto de responsabilidad social, y por lo tanto no se ve reflejada de manera explícita en los planes y programas (Entrevista-ST, 2016).

Al final, se pudo analizar el discurso oficial y el curriculum explícito de las IES del estado de Oaxaca con ayuda de los principios del análisis del discurso y así constatar si lo que se enuncia en el primero se refleja conceptual y semánticamente en el segundo, permitiendo con ello mostrar la necesidad de un esfuerzo colaborativo para hacer realidad los ideales educativos en el curriculum y así poder contribuir en efecto a mejorar las condiciones de la vida social del estado.

\section{Referencias}

Aguilera, Adriana y Puerto, Doria P. (2012). Crecimiento empresarial basado en la Responsabilidad Social. Pensamiento \& Gestión, 1-26 [consultado el 10 Ago 2016]. Disponible en: http:/ / www.redalyc.org/articulo.oa?id $=64623932002$

Alcoba, Jesús. (2013). Organización de los métodos de enseñanza en función de las finalidades educativas: El alineamiento curricular en Educación Superior. Profesorado. Revista de Curriculum y Formación de Profesorado, 17 [consultado el 12 Oct 2016]. Disponible en: http://www.redalyc.org/articulo. oa? id=56729527014

Arango, Olber E., Clavijo, Sandra J., Puerta, Isabel C., y Sánchez, José W. (2014). Formación académica, valores, empatía y comportamientos socialmente responsables en estudiantes universitarios. Revista de la educación superior, 43(169). [consultado el 04 Ago 2016]. Disponible en: http://www.scielo.org.mx/scielo. php?script=sci_arttext\&pid=S0185-27602014000100006\&lng=es\&tlng=es.

Biggs, John. (2006). Calidad del aprendizaje universitario. Madrid: Narcea

Bonilla, Elssy y Rodríguez, Penélope. (2005). Más allá del dilema de lométodos: la investigación en ciencias sociales. Bogotá: Norma 
Buenfil, Rosa N. (2010). Dimensiones ético políticas en educación desde el análisis político de discurso. Sinéctica, (35) [consultado el 27 Oct 2016]. Disponible en: http:/ / www.scielo.org.mx/scielo.php?script=sci_arttext\&pid=S1665109X2010000200010\&lng=es\&tlng=es

Cárdenas, María L., \& Rivera, José F. (2006). El análisis del discurso en el aula: una herramienta para la reflexión. Educere, 10(32) [consultado el 21 Oct 2016]. Disponible en: http://www.scielo.org.ve/scielo.php?script=sci_arttext\&pid=S131649102006000100006\&lng=es\&tlng=es.

Casarini, Martha. (2012). Teoría Curricular. México: Trillas

Castañeda, María T., Castro, Fancy y Mena, Carmen. (2012) Instrumentos para evaluar el curriculum formal en carreras pedagógicas. Panorama, 6 (10) [consultado el 15 Oct 2016]. Disponible en: http://journal.poligran.edu.co/index. php/panorama/article/viewFile/27/18

Comisión Estatal para la Planeación de la Educación Superior (2012). Programa de educación superior de Oaxaca 2011-2016. Oaxaca: Autor

Comisión Estatal para la Planeación de la Educación Superior (2015). Catálogo de la oferta de Posgrado en el Estado de Oaxaca 2015-2016. Oaxaca: Autor

Comisión Estatal para la Planeación de la Educación Superior (2016). Oferta de Educación Superior en el Estado de Oaxaca. Catálogo de Licenciaturas 2016-2017. Oaxaca: Autor

Consejo Nacional de Evaluación de la Política de Desarrollo Social (2016). Índice de rezago social 2015. México: Autor. [Consultado el 20 Jun 2016]. Disponible en: http://www.coneval.org.mx/Medicion/Documents/Indice_Rezago_Social_2015/Nota_Rezago_Social_2015_vf.pdf

Correa, Eleazar. (2009). Análisis del discurso educativo en México y su influencia en la conformación de la identidad de los adolescentes. Memoria del X Congreso Nacional de Investigación Educativa. Veracruz: Consejo Mexicano de Investigación Educativa, A.C.

Correa, Juan G. (2007). Evolución histórica de los conceptos de responsabilidad social empresarial y balance social. Revista Semestre Económico 10(20) [consultado el 08 Ago 2016]. Disponible en: http:/ / www.scielo.org.co/pdf/seec/ v10n20/v10n20a6.pdf

De Alba, Alicia. (1985). Evaluación de la congruencia interna de los planes de estudio; análisis de un caso. Revista de Educación Superior, 14 [consultado el 12 Oct 2016]. Disponible en: http:// publicaciones.anuies.mx/revista/54/2/3/ es/evaluacion-de-la-congruencia-interna-de-los-planes-de-estudio

De Sousa, Boaventura. (2007). La Universidad en el siglo xxi: para una reforma democrática y emancipadora de la Universidad. Buenos Aires: Miño y Dávila Editores

Duque, Yenni V., Cardona, Marleny y Rendón, Jaime A. (2013). Responsabilidad social empresarial: teorías, índices, estándares y certificaciones. Cuadernos de Administración, 29(1) [consultado el 10 Ago 2016]. Disponible en: http:// www.redalyc.org/articulo.oa?id=225029797009

Entrevista-ST. (2016). Secretario Técnico Licenciado Marcial Efrén Ocampo Ojeda. Oaxaca: Universidad Anáhuac Oaxaca, 15 de noviembre.

Fernández, Norberto. (2014). Universidad, sociedad y conocimiento reflexiones para el debate. Revista da Avaliação da Educação Superior, 3(19) [consultado el 20 Sep 2016]. Disponible en: http://www.scielo.br/pdf/aval/v19n3/08.pdf 
Fresan, María M. (2012). Acreditación y responsabilidad social universitaria. Apuntes para una segunda generación de indicadores. Memorias del Congreso Internacional de Acreditación y Certificación de programas e instituciones de educación superior 2012. Puerto Vallarta: copaes [consultado el 28 Sep 2016]. Disponible en: http://www.ibero.mx/sites/all/themes/ibero/descargables/estructuraynormatividad/Memorias_PDF_CIAC2012.pdf

Guerrero, María E. y Gómez, Diomedes A. (2013). Enseñanza de la ética y la educación moral, ¿permanecen ausentes de los programas universitarios? Revista Electrónica de Investigación Educativa. 15(1), [consultado el 20 Sep 2016]. Disponible en: http:/ / www.scielo.org.mx/pdf/redie/v15n1/v15n1a10.pdf

Guzmán, Juan C. (coord.); Arreola, Roxana L., Martínez, Oscar J., y Solís, Irene. (2013) Del curriculum al aula. Orientaciones y sugerencias para aplicar la rieb. México: Grao

Halliday, Michael A.K. (1994). El lenguaje como semiótica social. Bogotá: fce.

Halliday, Michael A.K. y Hasan, Ruqaiya. (1990). Language, context, and text: Aspects of language in a socio-semiotic perspective. Oxford: Oxford University Press.

Instituto Nacional de Estadística y Geografía (2015). Visor dinámico de bienestar. México: Autor, [consultado el 23 Jun 2016]. Disponible en: http:/ / www3.inegi.org.mx/sistemas/tubienestar/Indicadores.aspx

Iñiguez, Lupicinio. y Antaki, Charles. (1998) Análisis del Discurso. Revista Anthropos, 177 [consultado el 22 Oct 2016]. Disponible en: http:/ / www.psicol.unam. $\mathrm{mx} /$ Investigacion2/pdf/271_286.pdf

Leite, Betania. y Beltrán, José. (2012). Universidad y sociedad: la pertinencia de educación superior para una ciudadanía plena. Revista Lusófona de Educação, 21 [consultado el 20 Sep 2016]. Disponible en: http://www.redalyc.org/ pdf/349/34924585003.pdf

López, Carolina. (2015). El '98 cubano como vector dinámico en la emergencia de posturas en la intelectualidad argentina. Repositorio Institucional de la Universidad Nacional del Sur, [consultado el 10 Nov 2016]. Disponible en: http:/ / repositoriodigital.uns.edu.ar/handle/123456789/2032

López, Martha., Sañudo, Lya. y Maggi, Rolando E. (Coord.) (2013). Investigaciones sobre la investigación educativa 2002-2011. México: comie/ ANUIES

Martínez, Miquel. (2006) Formación para la ciudadanía y educación superior. En Revista Iberoamericana de Educación. 42, [consultado el 05 Ago 2016]. Disponible en: http:/ / rieoei.org/rie42a05.htm

Münch, Lourdes., Galicia, Emma y Jiménez, Susana. (2010). Administración y planeación de instituciones educativas. México: Trillas

Näsström, Gunilla., Henriksson, Widar. (2008). La alineación de estándares y evaluación. Un estudio teórico y empírico de métodos para la alineación. Electronic Journal of Research in Educational Psychology, 6, [consultado el 12 Oct 2016]. Disponible en: http:/ / www.redalyc.org/articulo.oa?id=293121916006

Navarro, Eva M. y Villarroel, Carlos. (2011). Universidad y sociedad: ¿Responsabilidades olvidadas? Editorial. Ingeniare, 19(2), [consultado el 20 Nov 2016]. Disponible en: http://www.scielo.cl/pdf/ingeniare/v19n2/art01.pdf

Olarte, Diana V. y Ríos, Leonardo A. (2015). Enfoques y estrategias de responsabilidad social implementadas en Instituciones de Educación Superior: Una revisión sistemática de la literatura científica de los últimos 10 años. Revista de la educación superior, 44(175), [consultado el 02 Ago 2016]. Dispo- 
nible en: http://www.scielo.org.mx/scielo.php?script=sci_arttext\&pid=S0185$27602015000300002 \& \operatorname{lng}=\mathrm{es} \& \ln \mathrm{l}=\mathrm{es}$.

Ortega, José. (2015). Misión de la Universidad. Madrid: Cátedra

Posner, George. (2005). Análisis del currículo. México: McGraw-Hill

Rodríguez, Teodosio E., Reyes, Manuel A, Peña, José A. (2009). Aproximación a un modelo para evaluar el currículo de la UPEL a partir de una propuesta de estructura curricular. Revista Universitaria de Investigación, 10(1), [consultado el 12 Oct 2016]. Disponible en: http://www.redalyc.org/articulo. oa?id $=41012305008$

Roldán, Leda M. (2005). Elementos para evaluar planes de estudio en la educación superior. Educación, 29 [consultado el 29 Oct 2016]. Disponible en: http:/ / www.redalyc.org/articulo.oa?id=44029111

Romero, Luis M. (2014). Programación didáctica: a modo de ejemplo. En Gómez, Inmaculada, y García, Francisco. J. (Coord.) Manual de didáctica, aprender a enseñar. Madrid: Pirámide, pp. 199 - 303

Sabaj, Omar. (2008). Tipos lingüísticos de análisis del discurso (ad) o un intento preliminar para un orden en el caos. Revista de lingüística teórica y aplicada, 46(2), [consultado el 10 Nov 2016]. Disponible en: https://dx.doi. org/10.4067/S0718-48832008000200007

Sayago, Sebastián; (2014). El análisis del discurso como técnica de investigación cualitativa y cuantitativa en las ciencias sociales. Cinta de Moebio, (49) [consultado el 10 Oct 2016]. Disponible en: http://www.redalyc.org/articulo. oa?id=10131417001

Secretaria de Educación Pública. (2013) Programa sectorial de educación 2013 2018. México: Autor

Soler, Sandra. (2011). Análisis crítico del discurso de documentos de política pública en educación. Forma y Función, 24(1) [consultado el 12 Oct 2016]. Disponible en: http:/ / www.redalyc.org/pdf/219/21922416005.pdf

Torruco, Uri, Díaz, Laura, Varela, Margarita y Martínez, Mildred. (2013). La entrevista, recurso flexible y dinámico. Investigación en Educación Médica, 2 [consultado el 14 Oct 2016]. Disponible en: http://www.redalyc.org/articulo. oa?id=349733228009

Urra, María E., Muñoz, Alejandra y Peña, Julio. (2013). El análisis del discurso como perspectiva metodológica para investigadores de salud. Enfermería Universitaria, 10 (2) [consultado el 20 Oct 2016]. Disponible en: http:/ / www. elsevier.es/es-revista-enfermeria-universitaria-400-articulo-el-analisis-deldiscurso-como-S1665706313726290\#bib0070

Vallaeys, François. (2006). Breve marco teórico de la responsabilidad social universitaria. Perú: Pontificia Universidad Católica del Perú [consultado el 15 Ago 2016]. Disponible en: http://rsuniversitaria.org/web/images/stories/BreveMarcoTeodelaResponsabilidadSocialUniv.pdf

Van Dijk, Teun. (2014). Análisis crítico del discurso. V Seminario ChileGloblaRedINCHE: Sociedad, poder y resistencia: Comprender Chile desde sus discursos. Barcelona, España. [consultado el 15 Oct 2016]. Disponible en: http://chilediscursos.migracionescomunicativas.cl/ 
Van Dijk, Teun A. y Mendizábal, Iván R. (1999). Análisis del discurso social y político. Quito: Abya-Yala.

Viteri, Jorge., Jácome, María B., Medina, Alberto y Piloto, Neydalis. (2012). Índice integral para evaluar la responsabilidad social universitaria en Ecuador. Ingeniería Industrial, 33 (3), [consultado el 05 Ago 2016]. Disponible en: http:/ / scielo.sld.cu/scielo.php?script=sci_arttext\&pid=S1815-59362012000300009\&lng $=\mathrm{es} \& \mathrm{t} \operatorname{lng}=\mathrm{es}$.

Zaldua, Alexei. (2006). El análisis del discurso en la organización y representación de la información-conocimiento: elementos teóricos. Acimed, 13 (3) [consultado el 24 Oct 2016]. Disponible en: http://bvs.sld.cu/revistas/aci/ vol14_3_06/aci03306.htm

Zúñiga, Marcela, Arévalo, Alejandra y Maggio, Maximiliano. (2014). Coherencia curricular y oportunidades para aprender Ciencias. Ciência E Educação (Bauru), 20(4) [consultado el 10 Sep 2016]. Disponible en: http:/ / www.scielo.br/ scielo.php?script=sci_arttext\&pid=S1516-73132014000400012\&lng=es\&nrm= iso\&tlng=en 
